\title{
ON THE GALOIS THEORY OF DIVISION RINGS
}

\author{
JOHN H. WALTER ${ }^{1}$
}

1. Throughout this paper, $K$ will represent a division ring and $L$ a galois division subring. We are interested in establishing a galois theory for the extension $K / L$ when $K / L$ is locally finite. In order to do this one must identify the galois subrings of $K$ containing $L$. An example given by Jacobson [4] shows that not every such division subring is galois. However, we obtain that each subring subject to a natural finiteness assumption is galois when the dimension $[K: H]_{l}$ $\leqq \boldsymbol{\aleph}_{0}$ where $H$ is the division subring left fixed by all inner automorphisms which leaves $L$ fixed. For these subrings we then obtain the usual theorems. We rely strongly on topological characterizations of the galois groups. In particular, we characterize the extensions with locally compact and complete galois groups. These results provide extensions and simplications of the recent results of Nagahara, Tominaga, and Nobusawa on division rings cited in the bibliography.

2. By $G(K / L)$, we mean the group of all automorphisms of $K$ leaving fixed a subring $L$; such a group will be called a galois group (sometimes this group has been called a total group of automorphisms). If $L^{\prime}$ is the fixed division ring of a group $G$ of automorphism of $K$, then $L^{\prime}$ is said to be a galois subring of $K$ and the extension $K / L^{\prime}$ is said to be galois; $L^{\prime}$ is necessarily a division ring.

The extension $K / L$ is said to be locally finite if every finite set of elements of $K$ is contained in a division ring whose dimension as a left $L$-module is finite. By $\left[L^{\prime}: L\right]_{l}$ and $\left[L^{\prime}: L\right]_{r}$ we mean the left and right dimensions of a division ring $L^{\prime}$ as, respectively, a left and right $L$-module. In cases where these are obviously the same, we make no distinction and write $\left[L^{\prime}: L\right]$. A finite extension $L^{\prime} / L$ is one where $\left[L^{\prime}: L\right]_{l}<\infty$.

On the group $G(K / L)$, we place the finite topology. Here a base for the neighborhoods of the identity consists of those groups of all automorphisms leaving fixed some finite subset of $K$. When $K / L$ is locally finite, they are of the form $G\left(K / L^{\prime}\right)$ where $\left[L^{\prime}: L\right]_{l}<\infty$. These subgroups are closed.

When $L^{\prime}$ is a division subring of $K$ containing $L$ and $G$ is a group of automorphisms, we denote by $G_{L^{\prime}}$ the set formed by the restrictions of the elements of $G$ to $L^{\prime}$. With the finite topology, this set is homeomorphic to the homogeneous space $G(K / L) / G\left(K / L^{\prime}\right)$. An extension

Received by the editors June 11, 1958 and, in revised form, March 9, 1959.

1 The author is a National Science Postdoctoral Fellow. 
$L^{\prime}$ of $L$ is normal in $K / L$ if $L^{\prime}$ is left invariant by the automorphisms of $G(K / L)$. If $L^{\prime}$ is a normal extension of $L$ in $K / L$ then $G_{L^{\prime}}$ is a group. The restriction mapping of $G$ onto $G_{L^{\prime}}$ is clearly continuous and is a homomorphism when $G_{L^{\prime}}$ is a group.

By $V_{K}\left(L^{\prime}\right)$, we mean the centralizer of a subring $L^{\prime}$ of $K$ in $K$. We will standardize our notation by always taking $T=V_{K}(L)$ and $H=V_{K}(T)$. We designate the center of a subring $L^{\prime}$ by $Z_{L^{\prime}}$; in particular, we set $Z_{K}=Z$.

We here mention some recent results of Nobusawa [11], of which we will make great use.

(N1) Let $K / L$ be galois and locally finite. Let $L^{\prime}$ be a division ring which is finite over $L$. Then $K / L^{\prime}$ is galois.

(N2) If $L^{\prime}$ is a division subring of $K$ containing $L,\left[V_{K}(L): V_{K}\left(L^{\prime}\right)\right]_{r}$ $\leqq\left[L^{\prime}: L\right]_{l}$. If $L^{\prime}=V_{K}\left(V_{K}\left(L^{\prime}\right)\right)$, then equality holds.

(N3) Let $K / L$ be galois and locally finite. Let $L^{\prime}$ be a division subring of $K$ containing $L$ and let $L^{\prime} H$ be the division subring generated by the elements of $L^{\prime}$ and $H$. Then $L^{\prime} H / L^{\prime}$ is outer galois and the restriction mapping

$$
\rho: G\left(L^{\prime} H / H\right) \rightarrow G\left(H / L^{\prime} H\right)
$$

is an isomorphism. Furthermore, if $\left[V_{K}(L): V_{K}\left(L^{\prime}\right)\right]_{r}<\infty$, then $L^{\prime} H$ $=V_{K}\left(V_{K}\left(L^{\prime}\right)\right)$. Also we will use freely the results of Jacobson $[5$, pp. 166-169] and Nobusawa [9].

3. In this section, we begin the development of the galois theory for the extensions $K / L$ which are locally finite and galois. These results are, in part, extensions and simplifications of results of Nagahara and Tominaga [7] and Nobusawa [11].

Proposition 1. Let $K / L$ be galois and locally finite. Then if $L^{\prime}$ is a division subring of $K$ containing $L,\left[H^{\prime}: H\right]_{l}=\left[L^{\prime}: H \cap L^{\prime}\right]_{l}=\left[T: T^{\prime}\right]_{r}$ where $T^{\prime}=V_{K}\left(L^{\prime}\right)$ and $H^{\prime}=L^{\prime} H$, the division subring of $K$ generated by $L^{\prime}$ and $H$. If $\left[H^{\prime}: H\right]_{l}<\infty$, then $H^{\prime}=V_{K}\left(V_{K}\left(L^{\prime}\right)\right)$.

Proof. By Nobusawa's theorem (N3), $H^{\prime} / L^{\prime}$ and $H / L^{\prime} \cap H$ are outer galois extension and the restriction mapping $\rho: G\left(H^{\prime} / L^{\prime}\right)$ $\rightarrow G\left(H / L^{\prime} \cap H\right)$ is an isomorphism. Now let $x_{1}, x_{2}, \cdots, x_{n}$ be $M$ independent elements of $L^{\prime}$ where $M=L^{\prime} \cap H$. We will show that they are $H$-independent elements of $H^{\prime}$ by applying a standard argument. Therefore, suppose that there exists a shortest expression

$$
\alpha_{1} x_{1}+\cdots+\alpha_{n} x_{n}=0
$$

with nonzero elements $\alpha_{i} \in H$. We may suppose that $n>1$ and that 
$\alpha_{1}=1$. Let $\sigma \in G\left(H^{\prime} / L^{\prime}\right)$. We have

$$
\underset{\alpha_{1} x_{1}}{\sigma}+\underset{\alpha_{2} x_{2}}{\sigma}+\cdots+\underset{\alpha_{n} x_{n}}{\sigma}=0 .
$$

Upon subtracting (2) from (1) and using the fact that $\alpha_{1}^{\sigma}=\alpha_{1}=1$, we obtain a shorter expression than (1). Thus unless $\alpha_{i}^{\sigma}=\alpha_{i}$, for $i=1,2, \cdots, n$ and all $\sigma \in G\left(H^{\prime} / L^{\prime}\right)$, we have a contradiction. This implies that $\alpha_{i} \in L^{\prime}$ and hence $\alpha_{i} \in L^{\prime} \cap H=M$ for $i=1,2, \cdots, n$. Finally the $M$-independence of the elements $x_{1}, x_{2}, \cdots, x_{n}$ gives a contradiction and (1) cannot exist. Thus the elements $x_{1}, x_{2}, \cdots, x_{n}$ are also $H$-independent. This implies that $\left[L^{\prime}: H \cap L^{\prime}\right]_{l} \leqq\left[H^{\prime}: H\right]_{l}$.

Now $\quad V_{K}\left(H^{\prime}\right)=V_{K}\left(L^{\prime}\right) \cap V_{K}(H)=V_{K}\left(L^{\prime}\right)=T^{\prime} ;$ also $V_{K}\left(H \cap L^{\prime}\right)$ $=V_{K}(H)=T$. Then by $(\mathrm{N} 2),\left[H^{\prime}: H\right]_{l}=\left[T: T^{\prime}\right]_{r} \leqq\left[L^{\prime}: H \cap L^{\prime}\right]_{l}$. This together with the preceding inequality proves the first statement of the proposition. The second statement then follows immediately from Nobusawa's theorem (N2) as Nobusawa pointed out.

The following is a generalization of part of [7, Lemma 9]; we use a modification of their argument.

Proposition 2. Let $K / L$ be galois and locally finite. Suppose that $L^{\prime}$ is a division subring of $K$ containing $L$ such that $\left[T: T^{\prime}\right]_{r}<\infty$. Then $K / L^{\prime}$ is locally finite.

Proof. We first assume that $H \supseteq L^{\prime} \supseteq L$. Let $F=\left\{y_{1}, y_{2}, \cdots, y_{m}\right\}$ be an arbitrary finite subset of $K$. Let $L(F), L^{\prime}(F)$, and $H(F)$ be the division subrings of $K$ generated by $F$ over the respective rings $L, L^{\prime}$ and $H$. Because $K / L$ is locally finite, $[L(F): L]_{l}<\infty$. Let $Q=L(F) \cap H$. Then $[Q: L]_{l}<\infty$. Let $x_{1}, x_{2}, \cdots, x_{n}$ be a $Q$-basis of $L(F)$ and let $q_{1}, q_{2}, \cdots, q_{p}$ be an $L$-basis of $Q$. By the proof of the previous theorem, $x_{1}, x_{2}, \cdots, x_{n}$ are $H$-independent. Therefore, they form an $H$-basis for $H(F)$.

Now $H / L$ is certainly locally finite and outer galois. Hence $G(H / L)$ is compact. Therefore, by Nobusawa [9], $H / L^{\prime}$ is locally finite. Let $M=L^{\prime} Q$. Since this is a division subring of $H$ generated over $L^{\prime}$ by the elements $q_{1}, q_{2}, \cdots, q_{p},\left[M: L^{\prime}\right]_{l}<\infty$.

Now consider the extension $H(F) / L(F)$. Since $H(F)=L(F) H$, $H(F) / L(F)$ is outer galois by $(\mathrm{N} 3)$. Since $Q=L(F) \cap H$, the restriction mapping $\rho: G(H(F) / L(F)) \rightarrow G(H / Q)$ is an isomorphism. Therefore, let $N$ be the fixed division subring of $\rho^{-1}(G(H / M))$. By the outer galois theory of Jacobson [5, p. 166] and Nobusawa [9], $G(H / M)$ is a closed subgroup of $G(H / Q)$ with fixed division subring $M$. But the fixed subring of $G(H(F) / N)=G(H / M)$ is $N \cap H$. Hence $N \cap H$ $=M$. Since $[L(F): L]_{l}<\infty$, it follows from (N2) and (N3) that $V_{K}\left(V_{K}(L(F))\right)=V_{K}\left(V_{K}(H(F))\right)=H(F)$. Then $\left[V_{K}\left(V_{K}(N)\right): H\right]_{l}$ $<\infty$. Thus from Proposition 1, $[N: M]<\infty$. 
Since $H(F) / L(F)$ is outer galois and locally finite and $H(F) \supseteq N$ $\supseteq L(F), H(F) / N$ is outer galois and hence locally finite. But then $\left[N: L^{\prime}\right]_{l}=[N: M]_{l}\left[M: L^{\prime}\right]_{l}<\infty$. Hence $H(F) / L^{\prime}$ is locally finite. Since $H(F) \supseteq L^{\prime}(F) \supseteq L^{\prime}, \quad\left[L^{\prime}(F): L^{\prime}\right]_{l}<\infty$. Thus $K / L^{\prime}$ is locally finite.

Finally we consider the case where $\left[T: T^{\prime}\right]_{r}<\infty$. Then by Proposition $1,\left[L^{\prime}: H \cap L^{\prime}\right]_{l}<\infty$. Since $K / H \cap L^{\prime}$ is locally finite, it follows that $K / L^{\prime}$ is also locally finite. This proves the proposition.

Using Theorem N1 and Propositions 1 and 2, we obtain the sufficiency of the following theorem.

Theorem 3. Let $K / L$ be galois and locally finite. Let $L^{\prime}$ be a division subring of $K$ containing $L$ such that $\left[L^{\prime}: H \cap L^{\prime}\right]<\infty$. Then a necessary and sufficient condition for $K / L^{\prime}$ to be galois is that $K / H \cap L^{\prime}$ is galois.

Proof. It only remains to prove the necessity of the theorem. Therefore, assume that $K / L^{\prime}$ is galois. Since $K / L$ is galois, $K / H$ is likewise galois. Let $G=G\left(K / L^{\prime}\right) G(K / H)$ be the subgroup generated by the elements of $G\left(K / L^{\prime}\right)$ and $G(K / H)$. Then clearly $H \cap L^{\prime}$ is contained in the fixed division subring of $G$.

Thus we see that the crucial remaining result to be sought is one that characterizes the galois subrings of $K$ contained in $H$ and containing $L$. This will be done in the next two sections.

4. Previously, Nagahara and Tominaga [7] developed a galois theory for division rings which satisfied four conditions. Nobusawa [11] recently showed that three of these conditions were a consequence of the condition that the galois extension $K / L$ be locally finite. The remaining condition was that $[T: Z]<\infty$. In their paper [7], Nagahara and Tominaga showed that if $\left[T: Z_{L}\right]<\infty$ and $K / L$ is locally finite, then a necessary and sufficient condition for $[T: Z]<\infty$ is that the galois group $G(K / L)$ be locally compact. We will improve this result in the following theorem.

Theorem 4. Let $K / L$ be locally finite. Then a necessary and sufficient condition for $[T: Z]<\infty$ is that the galois group $G(K / L)$ be locally compact.

Proof. Let $G(K / L)$ be locally compact. Then there exists a compact neighborhood of the identity, which is a subgroup $G\left(K / L^{\prime}\right)$ of $G(K / L)$ where $L^{\prime}$ is a finite extension of $L$. One may verify that $K / L^{\prime}$ is also locally finite. Let $L^{\prime \prime}$ be an arbitrary finite extension of $L^{\prime}$ in $K$. Then $G_{L^{\prime \prime}}\left(K / L^{\prime}\right)$ is a set of transformations of $L^{\prime \prime}$ on which we may place the finite topology. We have mentioned that the re- 
striction mapping $\rho: G\left(K / L^{\prime}\right) \rightarrow G_{L^{\prime \prime}}\left(K / L^{\prime}\right)$ is continuous. Thus $G_{L^{\prime \prime}}\left(K / L^{\prime}\right)$ is also compact. Since $\left[L^{\prime \prime}: L^{\prime}\right]_{l}<\infty$, the topology of $G_{L^{\prime \prime}}\left(K / L^{\prime}\right)$ is discrete; hence $G_{L^{\prime \prime}}\left(K / L^{\prime}\right)$ is finite. In other words, as every element of $K$ is contained in such a subring as $L^{\prime \prime}$, every element of $K$ has but a finite number of conjugates by elements of $G\left(K / L^{\prime}\right)$.

Let $T=V_{K}(L)$ and $T^{\prime}=V_{K}\left(L^{\prime}\right)$. By a result of Zelinsky, the centralizer $T^{\prime}$ is either infinite and $G\left(K / L^{\prime}\right)$ is outer or $T^{\prime}$ is a finite field (cf. Nobusawa [10]). If $G\left(K / L^{\prime}\right)$ is outer, then $T^{\prime}=Z$. In either case $T^{\prime} \supseteq Z$ and $\left[T^{\prime}: Z\right]<\infty$. By $(\mathrm{N} 2),\left[L^{\prime}: L\right]_{l} \geqq\left[T: T^{\prime}\right]_{r}$. Since $\left[L^{\prime}: L\right]$ $<\infty$, it follows that $\left[T: T^{\prime}\right]_{r}\left[T^{\prime}: Z\right]_{r}=[T: Z]<\infty$.

To prove the converse, we use the notation of the previous paragraphs. Set $H=V_{K}(T)$. We assume that $[T: Z]<\infty$. Then by $[1$, Theorem 13], $[K: H]_{l}=[T: Z]<\infty$. Let $x_{1}, x_{2}, \cdots, x_{n}$ be a left $H$-basis for $K$ and let $L^{\prime}$ be the division ring generated by the elements $x_{1}, x_{2}, \cdots, x_{n}$ over the ring $L$. If $L^{\prime} H$ is the division subring of $K$ generated by $L^{\prime}$ and $H$, then $L^{\prime} H=K$. Thus $Z=V_{K}\left(L^{\prime} H\right)$ $=V_{K}\left(L^{\prime}\right) \cap V_{K}(H)=T^{\prime} \cap T \supseteq Z$. Since $T \supseteq T^{\prime}$, it follows that $T^{\prime}=Z$. Thus $G\left(K / L^{\prime}\right)$ is outer. Since $\left[L^{\prime}: L\right]_{l}$ is locally finite, $G\left(K / L^{\prime}\right)$ is compact by a result of Jacobson $[5$, p. 166]. Thus $G(K / L)$ is locally compact. This completes the proof.

5. Recently Nagahara and Tominaga [8], showed that if $[K: H]$ $\leqq \boldsymbol{\aleph}_{0}$ and $K / L$ galois and locally galois then $G_{H}(K / L)=G(K / L)$. This enables them to conclude that every division subring of $H$ containing $L$ is galois in $K$. In Theorem 6 , we will obtain this result with the assumption that $K / L$ is locally galois replaced by the assumption that $K / L$ is locally finite.

Let $K / L$ be a galois and locally finite extension. Denote by $G^{\prime}(K / L)$ the set of all isomorphisms of $K$ into itself leaving $L$ fixed. On this set place the finite topology. It follows directly from Jacobson's extension theorem [5, p. 162] that every element of $G^{\prime}(K / L)$ agrees on each finite subset $F \subset K$ with an element of $G(K / L)$. Hence $G(K / L)$ is dense in $G^{\prime}(K / L)$. In parallel with the situation with the group $G(K / L)$, it is easily seen that a base for the neighborhoods of an element $\sigma \in G(K / L)$ consists of the sets $\sigma G^{\prime}\left(K / L^{\prime}\right)$ where $L^{\prime} / L$ is a finite extension. Since $G(K / L)$ is dense in $G^{\prime}(K / L)$, these sets also form a neighborhood basis for $G^{\prime}(K / L)$. Just as with topological groups, $G^{\prime}(K / L)$ is a uniform space when we take for the uniformity on $G^{\prime}$, the subsets of $G^{\prime} \times G^{\prime}$ consisting of elements $\left(\tau, \tau^{\prime}\right)$ where $\tau$ and $\tau^{\prime}$ agree with some fixed isomorphism into $K$ of a division ring $L^{\prime} \supset L$ such that $\left[L^{\prime}: L\right]<\infty$. These are the sets $\left(\tau, \tau^{\prime}\right)$ where $\tau \in \sigma G^{\prime}\left(K / L^{\prime}\right)$ 
and $\tau^{\prime} \in \sigma G^{\prime}\left(K / L^{\prime}\right)$ for some fixed $\sigma \in G(K / L)$ and fixed finite exten$\operatorname{sion} L^{\prime} / L$.

Again denote by $G_{L^{\prime}}(K / L)$ the set of restrictions of elements of $G^{\prime}(K / L)$ to $L^{\prime} \supset L$.

Proposition 5. Let $K / L$ be galois and locally finite. Let $[K: H]$ $=\aleph_{0}$. Then $G_{H}^{\prime}(K / L)=G(H / L)$.

Proof. Let $\mathcal{F}$ be the set of all finite subsets of $K$. Let $\mathfrak{F}$ $=\{H(F) \mid F \in \mathcal{F}\}$; clearly $\mathfrak{F}$ is a directed set and $U\{H(F) \mid F \in \mathcal{F}\}=K$. Form the sets $G^{\prime}(H(F) / L)$ of isomorphisms of $H(F)$ into $K$ leaving $L$ fixed. Using the restriction homomorphisms $\rho_{\alpha \beta}: G^{\prime}\left(H\left(F_{\beta}\right) / L\right)$ $\rightarrow G^{\prime}\left(H\left(F_{\alpha}\right) / L\right)$ where $F_{\beta} \subseteq F_{\alpha}$ we form the inverse limit $G^{\prime}$ $=\lim G^{\prime}\left(H\left(F_{\alpha}\right) / L\right)$. Let $\rho_{\alpha}$ be the mapping of $G^{\prime}$ onto the component $G^{\prime}\left(H\left(F_{\alpha}\right) / L\right)$. Then by setting for $\sigma \in G^{\prime}$ and $x \in K, \sigma x$ $=\left(\rho_{\alpha} \sigma\right) x x \in H\left(F_{\alpha}\right)$, it is easy to see that $\sigma$ determines an element of $G^{\prime}(K / L)$. Hence $G^{\prime} \subseteq G^{\prime}(K / L)$. On the other hand, an element of $G^{\prime}$ whose components in $G\left(H\left(F_{\alpha}\right) / L\right)$ are determined as the restrictions to $H\left(F_{\alpha}\right)$ of an element of $G^{\prime}(K / L)$ is easily seen to be in $G^{\prime}$. Hence $G^{\prime}(K / L)=\lim G^{\prime}\left(H\left(F_{\alpha}\right) / L\right)$. We will establish the proposition by making use of this decomposition.

Set $L^{\prime}=L\left(F_{0}\right)$ and $H^{\prime}=H\left(F_{0}\right)$ where $F_{0}$ is an arbitrary finite set. Then $L^{\prime} / L$ and $H^{\prime} / H$ are both finite extensions by Proposition 2. Furthermore, $V_{K}\left(H^{\prime}\right)=V_{K}\left(H L^{\prime}\right)=V_{K}(H) \cap V_{K}\left(L^{\prime}\right)=V_{K}(L) \cap V_{K}\left(L^{\prime}\right)$ $=V_{K}\left(L^{\prime}\right)$ as $L^{\prime} \supseteq L$. This means, first of all, that $V_{H^{\prime}}\left(L^{\prime}\right)=V_{K}\left(L^{\prime}\right) \cap H^{\prime}$ $=V_{H^{\prime}}\left(H^{\prime}\right)$. Hence $G\left(H^{\prime} / L^{\prime}\right)$ contains only outer automorphisms. Also we see that $V_{K}\left(V_{K}\left(L^{\prime}\right)\right)=H^{\prime}$. Hence $H^{\prime}$ is left invariant by the elements of $G\left(K / L^{\prime}\right)$.

We claim that $H^{\prime}$ is left invariant by the elements of $G^{\prime}\left(K / L^{\prime}\right)$; that is, if $\sigma \in G^{\prime}\left(K / L^{\prime}\right)$, then $\sigma H^{\prime} \subseteq H^{\prime}$. Indeed, if this were not true, then there would exist a finite subset $F \subset H^{\prime}$ such that $\sigma F$ is not contained in $H^{\prime}$. Now $K / L^{\prime}$ is galois by Theorem $\mathrm{N} 1$ and locally finite since $\left[L^{\prime}: L\right]_{l}<\infty$. Hence by Jacobson's extension theorem, there exists an extension $\sigma^{\prime}$ of the restriction of the isomorphism $\sigma$ on the finite extension $L^{\prime}(F) / L^{\prime}$ to an automorphism of $K$. But we have seen then that $\sigma^{\prime} H^{\prime}=H^{\prime}$; hence $\sigma L^{\prime}(F)=\sigma^{\prime} L^{\prime}(F) \subseteq H^{\prime}$, which is a contradiction. This argument also shows that the group $G\left(H^{\prime} / L^{\prime}\right)$ is dense in $G^{\prime}\left(H^{\prime} / L^{\prime}\right)$, the set of isomorphisms of $H^{\prime}$ into $K$.

Next we claim that $G^{\prime}\left(H^{\prime} / L^{\prime}\right)$ is locally compact in the finite topology and that $G^{\prime}\left(H^{\prime} / L^{\prime}\right)=G\left(H^{\prime} / L^{\prime}\right)$. As argued before, $H^{\prime} / L^{\prime}$ is galois and locally finite. Furthermore, $G\left(H^{\prime} / L^{\prime}\right)$ contains only outer automorphisms. It then results from $\left[5\right.$, p. 166] or [9] that $G\left(H^{\prime} / L^{\prime}\right)$ is compact. Hence $G\left(H^{\prime} / L^{\prime}\right)$ is closed and dense in $G^{\prime}\left(H^{\prime} / L^{\prime}\right)$. This 
means that $G^{\prime}\left(H^{\prime} / L^{\prime}\right)=G\left(H^{\prime} / L^{\prime}\right)$ is compact. This is then still true for $G^{\prime}\left(H^{\prime} / L^{\prime \prime}\right)$ when $H^{\prime} \supset L^{\prime \prime} \supset L^{\prime}$. Thus the sets $\left\{\sigma G^{\prime}\left(H^{\prime} / L^{\prime \prime}\right)\right\}$ where $L^{\prime \prime} \supset L^{\prime}$ and $\left[L^{\prime \prime}: L\right]<\infty$ and $\sigma \in G\left(H^{\prime} / L\right)$ form a compact neighborhood basis for $G^{\prime}\left(H^{\prime} / L\right)$.

Now let $H_{1}^{\prime} \supset H_{2}^{\prime} \supset H$ and $H_{1}^{\prime} / H$ be a finite extension. Let $\rho: G^{\prime}\left(H_{1}^{\prime} / L\right) \rightarrow G^{\prime}\left(H_{2}^{\prime} / L\right)$ be the restriction homomorphism. Clearly $\rho$ is uniformly continuous. We wish to show that $\rho$ is open and onto. Let $G^{\prime}\left(H_{1}^{\prime} / L_{1}^{\prime}\right)=G\left(H_{1}^{\prime} / L_{1}^{\prime}\right)$ be a compact open subgroup of $G^{\prime}\left(H_{1}^{\prime} / L\right)$. Then $\rho G^{\prime}\left(H_{1}^{\prime} / L\right)$ is a compact subgroup of $G^{\prime}\left(H_{2}^{\prime} / L\right)$. On the other hand, for any element $x \in H_{2}^{\prime}$ such that $x \in L_{1}^{\prime} \cap H_{2}^{\prime}$, there exists an isomorphism $\sigma \in G^{\prime}\left(H_{2} / L_{1}^{\prime}\right)$ such that $\sigma x \neq x$. Hence the division ring left fixed by all the elements of $\rho G^{\prime}\left(H_{1}^{\prime} / L_{1}^{\prime}\right)$ is $L_{1}^{\prime} \cap H_{2}^{\prime}$. Since $\rho G^{\prime}\left(H_{1}^{\prime} / L_{1}^{\prime}\right)$ is a group, $H_{2}^{\prime} / L_{1}^{\prime} \cap H_{2}^{\prime}$ is galois. Since $L_{1}^{\prime} \supset L_{1}^{\prime} \cap H_{2}^{\prime}$, [ $\left.L_{1}^{\prime} \cap H_{2}^{\prime}: L\right]_{l}<\infty$, and $H_{2}^{\prime} / L_{1}^{\prime} \cap H_{2}^{\prime}$ is locally finite. As argued previously, $\rho G^{\prime}\left(H_{1}^{\prime} / L_{1}^{\prime}\right)$ is dense in the open and closed set $G^{\prime}\left(H_{2}^{\prime} / L_{1}^{\prime} \cap H_{2}^{\prime}\right)$. Hence $\rho G^{\prime}\left(H_{1}^{\prime} / L_{1}^{\prime}\right)=G^{\prime}\left(H_{2}^{\prime} / L_{1}^{\prime} \cap H_{2}^{\prime}\right)$ is open and one may easily verify that $\rho$ is open.

Just as in the case of topological groups, it follows that because $G^{\prime}\left(H_{1}^{\prime} / L\right)$ and $G^{\prime}\left(H_{2}^{\prime} / L\right)$ are locally compact, they are complete. But then the images of $G^{\prime}\left(H_{1}^{\prime} / L\right)$ by uniformly continuous open mappings are complete and hence closed. Thus $\rho G^{\prime}\left(H_{1}^{\prime} / L\right)$ is a closed subset of $G^{\prime}\left(H_{2}^{\prime} / L\right)$. But the restriction of any element $\sigma$ of $G^{\prime}\left(H_{2}^{\prime} / L\right)$ to a finite subset $F$ of $K$ defines an isomorphism of $L(F)$ into $K$. By Jacobson's extension theorem, this may be extended to an automorphism of $K$ and thus certainly to an isomorphism of $H_{1}^{\prime}$ into $K$. Thus $\sigma$ agrees on $F$ with some element of $\rho G^{\prime}\left(H_{1}^{\prime} / L\right)$. Hence $\rho G^{\prime}\left(H_{1}^{\prime} / L\right)$ is dense in $G^{\prime}\left(H_{2}^{\prime} / L\right)$ and $\rho G^{\prime}\left(H_{1}^{\prime} / L\right)=G^{\prime}\left(H_{2}^{\prime} / L\right)$; thus $\rho$ is onto.

We next show that the restriction homomorphism $\rho_{0}: G^{\prime}(K / L)$ $\rightarrow G^{\prime}(H / L)$ is onto. Because $[K: H]_{l} \leqq \boldsymbol{\aleph}_{0}$, there exists a cofinal subset $\left\{H_{i} \mid i=1,2, \cdots\right\} \subseteq \mathcal{J C}$ such that $H_{i} / H$ is a finite extension and $K=\bigcup_{i=1}^{\infty} H_{i}$. Furthermore, each restriction homomorphism $\rho_{\alpha \beta}: G^{\prime}\left(H\left(F_{\beta}\right) / L\right) \rightarrow G^{\prime}\left(H\left(F_{\alpha}\right) / L\right)$ has been shown to be onto. Under these conditions, it follows from [3] (cf. also [2, Chapter 8]) that $\rho_{0}$ is onto. Since we have already shown that $G^{\prime}(H / L)=G(H / L)$, the proof is complete.

Theorem 6. Let $K / L$ be galois and locally finite. Let $[K: H]_{l} \leqq \boldsymbol{\aleph}_{0}$. Then if $L^{\prime}$ is a division subring of $H$ containing $L, K / L^{\prime}$ is galois.

Proof. We here refer to Jacobson [5, Chapter VI] in order to use a theorem of Nakayama. There the galois theory of a ring $\mathbb{R}$ of all linear transformations of a left vector space $\mathfrak{M}$ over a division 
ring $\Delta$ is discussed. The elements of $\mathbb{R}$ are taken to be right operators on $\mathfrak{M}$. A subring $\mathfrak{A}$ of $\mathfrak{R}$ is said to be weakly galois if it is the centralizer of a set $\mathfrak{U}$ of semilinear transformations of $\mathfrak{M}$ over $\Delta$. The elements of $\mathfrak{U}$ are to be considered as left operators on $\mathfrak{M}$.

We set $\mathfrak{M}=K$ and take for $\mathfrak{R}$ the division ring $K_{R}$ of right multiplications induced by elements of $K$. We take $\Delta=K_{L}$ the ring of left multiplications induced by the elements of $K$. Let $\mathfrak{U}=\oplus \Delta \sigma$ where the sum is over all isomorphisms $\sigma \in G^{\prime}\left(K / L^{\prime}\right)$. Let $\mathfrak{A}$ be the centralizer of $\mathfrak{U}$ in the ring of all additive endomorphisms of $\mathfrak{M}$. Then $\mathfrak{A}$ is weakly galois. Since $\mathfrak{U} \supset \Delta, \mathfrak{A}$ is a division subring of $\mathfrak{R}=K_{R}$. More than this $\mathfrak{A}$, we claim, is the division subring $L_{R}^{\prime}$ induced by $L^{\prime}$. Indeed, $\mathfrak{A}$ is determined as the fixed ring of $G^{\prime}\left(K / L^{\prime}\right)$. But it easily follows that $L^{\prime}$ is the fixed ring of $G\left(H / L^{\prime}\right)$. Thus from Proposition $5, L^{\prime}$ is the fixed ring of $G_{H}^{\prime}\left(K / L^{\prime}\right)$ and thus of $G^{\prime}\left(K / L^{\prime}\right)$. Hence $\mathfrak{A}=L_{R}^{\prime}$.

We wish to show that $\mathfrak{A}$ is a galois subring of $\mathfrak{R}$; that is, we wish to establish that $\mathfrak{U}$ is generated by invertible semilinear transformations. To do this, observe first that the four conditions of [5, Proposition 6.11 .2$, p. 146] are satisfied. Indeed, the first two are trivially true. That the last two hold follows from the fact that any subring of the ring of additive endomorphisms of $\mathfrak{M}$ which contains $\Delta$ is an irreducible and, hence, homogeneous ring of transformations. Also it follows from this proposition that $\mathfrak{R} \Delta$ is an irreducible and, hence, homogeneous ring of transformations. But then from Nakayama's theorem [5, Proposition 6.11 .3 , p. 147], it follows that $\mathfrak{A}$ is a galois subring of $\mathfrak{l}=K_{R}$. Since $\mathfrak{A}=L_{R}^{\prime}, L^{\prime}$ is a galois subring of $K$.

6. We wish to characterize the subgroups of a galois group which are themselves galois groups. We say that a group of automorphisms is regular if it contains all the inner automorphisms which are generated by the elements of the centralizer of its fixed division subring. We now prove the following result. ${ }^{2}$

Proposition 7. Let $K / L$ be locally finite and galois. Let $G^{\prime}$ be a regular closed subgroup of $G(K / L)$, with fixed division subring $L$. Then $G^{\prime}=G(K / L)$.

Proof. Set $G=G(K / L)$. Let $L^{\prime}$ be an arbitrary finite extension of $L$. We show that $G_{L^{\prime}}=G_{L^{\prime}}^{\prime}$. Clearly $G \supseteq G^{\prime}$; so $G_{L^{\prime}} \supseteq G_{L^{\prime}}^{\prime}$. But by Jacobson's extension theorem [2, p. 162], it follows from the regularity of $G_{L^{\prime}}^{\prime}$ that every isomorphism of $L^{\prime}$ into $K$ contained in $G_{L^{\prime}}$ can

${ }^{2}$ I wish to thank Professors Rosenberg and Zelinsky for pointing out that our result actually follows directly from Jacobson's extension theorem as above and for their comments in general. 
be extended to an element of $G^{\prime}$. Hence $G_{L^{\prime}}^{\prime} \supset G_{L^{\prime}}$. Thus $G$ and $G^{\prime}$ agree on every finite subset of $K$ and, therefore, their closures are identical. By the hypothesis of the proposition $G=G^{\prime}$.

From this one may conclude that the regular closed subgroups of $G(K / L)$ are the galois groups $G\left(K / L^{\prime}\right)$ of the galois extensions $K / L^{\prime}$, where $L^{\prime} \supset L$. Given a closed subgroup $G$ of $G(K / L)$, let $L(G)$ be its fixed subring. Let $H(G)=V_{K}\left(V_{K}(L(G))\right)$ and $H=V_{K}\left(V_{K}(L)\right)$. Then if $[H(G): H]_{l}<\infty, G$ is said to have finite degree. Likewise, a division ring $L^{\prime} \supset L$ is said to have finite degree if $\left[T: T^{\prime}\right]_{r}<\infty$.

Theorem 8. Let $K / L$ be a galois and locally finite extension and let $[K: H] \leqq \aleph_{0}$. Then there exists a one-to-one correspondence between the regular closed subgroups $G$ of finite degree and the division subrings $L^{\prime}$ of finite degree of $K$ containing $L$. Furthermore, $G(K / L(G))=G$ and $L\left(G\left(K / L^{\prime}\right)\right)=L^{\prime}$.

This theorem establishes the galois theory we promised; its proof is direct from Theorems 3, 6 and Proposition 7.

7. Remarks. Under stronger hypotheses, of course, it is much easier to establish the results of this paper. For example, if one assumes that $G(K / L)$ is locally compact, then, as we have argued, $G(K / L)$ is complete and the restriction homomorphism $\rho: G(K / L)$ $\rightarrow G(H / L)$ is uniformly continuous and open; thus $\rho G(K / L)$ is closed in $G(H / L)$. Since $\rho G(K / L)$ is dense in $G(H / L), \rho G(K / L)=G_{H}(K / L)$ $=G(H / L)$. This short argument can replace Proposition 5 and Theorem 6.

Another interesting result is obtained by noticing that the proof of Proposition 5 establishes the fact that $G^{\prime}(K / L)$ is the inverse limit of complete topological spaces and hence is complete whenever $K / L$ is galois and locally finite. Since Jacobson's extension theorem shows that $G(K / L)$ is dense in $G^{\prime}(K / L)$, it follows that a necessary and sufficient condition for all isomorphisms of $K$ leaving $L$ fixed to be automorphisms is that $G(K / L)$ be complete in its uniform topology. That $G^{\prime}(K / L)$ is complete also follows simply from the fact that $G^{\prime}(K / L)$ is a closed subset of the space $K^{K}$ of functions of $K$ into $K$. When $K$ is discrete, $K^{K}$ is complete.

The case of locally finite dimensional extensions has been considered frequently in the literature. An extension $K / L$ is said to be locally finite dimensional (1.f.d.) if for every finite subset $F \subset K$, $\left[L\left(F^{G}\right): L\right]_{l}<\infty$ where here $F^{G}$ denotes the set of images of elements of $F$ by the elements of $G$.

Now if $K / L$ is galois and l.f.d., there exists a directed set $\mathscr{N}$ of 
finite extensions $N / L$ such that each $N \in \mathfrak{N}$ is normal in $K / L$. But since $G(N / L)$ is discrete $G_{N}(K / L)=G(N / L)$. Again using the restriction homomorphisms, form the inverse $\operatorname{limit} \lim G(N / L)$ where $N \in \mathfrak{N}$. As we argued in a similar situation in the proof of Proposition 5, $G(K / L)=\lim G(N / L)$. Since each group $G(N / L)$ is discrete, it is complete. Hence $G(K / L)$ is complete. We have proved the following proposition.

Proposition 9. Let $K / L$ be galois and locally finite dimensional. Then $G(K / L)$ is complete in its uniform topology.

\section{BIBLIOGRAPHY}

1. E. Artin and R. G. Whaples, The theory of simple rings, Amer. J. Math. vol. 65 (1943) pp. 87-107.

2. S. Eilenberg and N. Steenrod, Foundations of algebraic topology, Princeton, 1952.

3. L. Henkin, A problem on inverse mapping systems. Proc. Amer. Math. Soc. vol. 1 (1950) p. 224.

4. N. Jacobson, A note on two dimensional division ring extensions, Amer. J. Math. vol. 77 (1955) pp. 593-595.

5. - Structures of rings, Amer. Math. Soc. Colloquium Publications, vol. 37, Providence, 1956.

6. M. Moriya, T. Nagahara and H. Tominaga, $A$ note of galois theory of division rings, Math. J. Okayama Univ. vol. 7 (1957) pp. 83-88.

7. T. Nagahara and H. Tominaga, On the galois theory of division rings, Math. J. Okayama Univ. vol. 6 (1956) pp. 1-21.

8. - On the galois theory of division rings, II, Math. J. Okayama Univ. vol. 7 (1957) pp. 169-173.

9. N. Nobusawa, An extension of Krull's galois theory of division rings, Osaka Math. J. vol. 7 (1955) pp. 1-6.

10. - On compact galois groups of division rings, Osaka Math. J. vol. 8 (1956) pp. 43-49.

11. $-A$ note on galois extensions of division rings, Math. J. Okayama Univ. vol. 7 (1957) pp. 179-185.

UNIVERSITY OF WASHINGTON AND INSTITUTE FOR AdVANCED StUdy 